\title{
Brachytherapy in head and neck malignancies: Indian Brachytherapy Society (IBS) recommendations and guidelines
}

\author{
Rajendra Bhalavat, MD!, Ashwini Budrukkar, MD, DNB², Sarbani Ghosh Laskar, MD², Dayanand Sharma, MD³, \\ Ashutosh Mukherij, MD4, Manish Chandra, DNB!, Umesh Mahantshetty, MD², Vibhay Pareek, DNB 5 , \\ Pratibha Bauskar, M.Pys, RSO', Sonali Saraf, MD 6 \\ 'Department of Radiation Oncology, Jupiter Lifelines Hospital, Thane, India, ${ }^{2}$ Department of Radiation Oncology, Tata Memorial Hospital, \\ Parel, India, ${ }^{3}$ Department of Radiation Oncology, All India Institute of Medical Sciences, New Delhi, India, ${ }^{4}$ Department of Radiation Oncology, \\ Mahamana Pandit Madanmohan Malviya Cancer Center and Homi Bhabha Cancer Hospital, Varanasi, India, ${ }^{5}$ Department of Radiation \\ Oncology, National Cancer Institute, AllMS, New Delhi, India, 'Department of Oncoanesthesia, Jupiter Lifelines Hospital, Thane, India
}

\begin{abstract}
Purpose: Brachytherapy (BT) forms major treatment modality in squamous cell carcinoma of head and neck cancers (HNC). However, there is a dearth of literature and guidelines for the use in various indications. High-dose-rate brachytherapy (HDR-BT) in Indian scenario is an important treatment modality, and the recommendations in this guidelines aim to provide the necessary recommendations for the use of HDR-BT for uniform application across the country in patients with HNC.

Material and methods: A panel consisting of members of the Indian Brachytherapy Society (IBS), based on their clinical experience was invited. The process involved defining important steps, precautions, target volumes and indications, thorough literature review, and discussion with fellow members. The guidelines were established and formulated the recommendations for HDR-BT based on available evidences and individual experience for sites, relevant to Indian settings.

Results: The IBS recommends the use of HDR brachytherapy as a part of treatment of head and neck tumors. The scope of these guidelines and recommendations included practical suggestions, ensuring efficient use of brachytherapy treatment as radical with external beam radiotherapy (EBRT) boost, palliative and adjuvant as definitive, or re-radiation as salvage for HNC in India. The IBS has made specific site-wise recommendations for previously untreated and recurrent HNC patients on their selection criteria, implant techniques, target volume definition, and HDR treatment parameters, such as time, dose rate, total dose, and fractionation schedules. Limited experience exists with HDR-BT in patients with head and neck cancers in India and across the globe.

Conclusions: IBS provided a consensus statement and guidelines for the head and neck brachytherapy and believed that these recommendations will overcome the fear of practicing radiation oncologists. This should generate interest amongst students and will help radiation oncologists all across the country to use the art of brachytherapy carefully in HNC patients, with better curative and salvage options.
\end{abstract}

J Contemp Brachytherapy 2020; 12, 5: 501-511 DOI: https://doi.org/10.5114/jcb.2020.100385

Key words: head and neck malignancies, HDR brachytherapy, guidelines, recommendations.

\section{Purpose}

Head and neck squamous cell carcinoma (HNC) accounts for one-third of oral cancer cases in the world and constitute $30 \%$ of all cancers in India [1]. India has a distinct demographic profile, risk factors, food habits, personal, and family histories that contribute to the HNC being the leading public health problem with oral cavity and oropharynx, including most of HNCs in India [2].
Brachytherapy alone or combined with external beam radiotherapy provides well-established indications in HNC $[3,4]$. A comparison between surgery and radiotherapy has never been determined in a randomized trial setting and both approaches have their limitations $[5,6]$. However, brachytherapy offers the advantage of rapid exponential peripheral fall-off around planning treatment volume (PTV) and provides a localized high-dose 
radiation as well as reduces damage to adjacent normal tissues $[7,8]$.

The majority of patients with HNC have a locally advanced disease (LAD) at diagnosis, and multimodality treatment remains the mainstay of treatment involving radiotherapy in combination with surgery, chemotherapy, and immunotherapy. Radiotherapy (concurrent radiochemotherapy) consists of external beam radiotherapy (EBRT), with weekly platinum-based chemotherapy for 6-7 weeks as a radical curative intent. The selection of the most appropriate strategy for a primary malignant tumor is influenced by anatomical location, extent, and histopathology of neoplasm as well as host factors, such as general condition, comorbidities, socioeconomic status, and geographical location. The goal of any treatment besides survival is the preservation of organs and/or function, with acceptable post-treatment quality of life (QoL). Dose intensification is necessary for better local and regional control of disease. EBRT alone is associated with undesirable early and late toxicity, despite the recent advances in technology and treatment delivery. Considerable experience and insight gained in extensive clinical research and widespread clinical practice with low-dose-rate brachytherapy (LDR-BT), pulsed-dose-rate brachytherapy (PDR-BT) $[9,10,11,12,13]$, and recently, high-dose-rate brachytherapy (HDR-BT), have demonstrated similar advantages in many sites, including uterine cervix, prostate, sarcoma, and breast malignancies. However, it has not gained widespread acceptance among radiation oncologists (ROs) for HNC.

Radiation oncologists are well aware of the role of brachytherapy in early-stage as a sole treatment or in combination with surgery or EBRT for suitable sites $[14,15,16$, $17,18,19]$. There are various guidelines for treatment of HNC comprising brachytherapy, but these guidelines are

Table 1. Indications and various brachytherapy techniques

\begin{tabular}{ll} 
Technique & \multicolumn{1}{c}{ Subsites } \\
\hline Interstitial & Oral cavity: \\
& - lip \\
& - buccal mucosa \\
& - oral tongue \\
& - floor of mouth \\
& Oropharynx: \\
& - base of tongue/vallecula \\
& - soft palate \\
& - tonsil \\
& - epiglottis \\
& Nasopharynx/external auditory canal \\
& (EAC) \\
\hline Intraluminal & External ear \\
& Nasal vestibule \\
& Hard palate \\
Surface mould & Skin of face (BCC/SCC) \\
(SM) & Hard palate + soft palate \\
& Tumors of nasal vestibule
\end{tabular}

primarily designed and applicable for the western world. In India, we need to consider the unique cultural background, different patterns of diseases, specific healthcare system, delay in diagnosis, problems in accessing treatment facilities, and logistic differences, and thereby, provide comprehensive guidelines addressing all those issues. Radiation oncologists in India came together and created the Indian Brachytherapy Society (IBS) 15 years ago to practice, teach, conduct workshops, conferences, researches, and promote brachytherapy as well as formulate protocols and guidelines for its members in various sites. The IBS has taken a call to ask, collect, and summarize the experience of some of the ROs, who practice brachytherapy in HNC for all or specific sites, and establish consensus recommendations to benefit all those who want to treat HNC patients with brachytherapy.

\section{Methodology for consensus guidelines}

A panel of working members from the IBS, who practice HNC brachytherapy and treat a relatively large number of HNC patients was created. The aim of this guidelines and recommendations was to review, discuss, and propose some practical aspects to ensure a minimum standard of brachytherapy use as a radical treatment or in combination with radiation therapy for HNC in India. The process involved defining important steps, detailed literature review and discussion, and guidelines based on available evidence, and individual experience and its relevance to Indian settings. These consensus recommendations with the spectrum of clinical practice in various subsites and limited literature, provided by a panel of experts will be useful for brachytherapy students and BT beginners. The recommendations covered general aspects of HNC and brachytherapy under various titles, including: 1) patients' selection, pre-treatment workup, and patient care, 2) treatment strategy, 3) target definition, 4) implant techniques, 5) HDR dose and doserate prescription, 6) treatment planning and reporting, 7) treatment monitoring 8) catheter removal, and 9) posttreatment patient care and follow-up.

\section{Introduction to interstitial brachytherapy}

Interstitial placement remains the main method for various sites of $\mathrm{HNC}$, although surface mold and intraluminal brachytherapy may be used with respect to the involved site (Table 1). The use of brachytherapy poses a challenge in the head and neck area because it is an anatomical structure of many subsites, having distinct anatomical shape and curvature, with close proximity to vital structures. This makes needle insertions and application difficult, requiring care of important vessels and vital structures leading to non-uniformity of implant planes. Brachytherapy in HNC sites is generally multi-planar; however, a single-plane may be sufficient enough for early T1 lesions of the lip, buccal mucosa, and soft palate lesions. A single-plane for post-operative/peri-operative (tumor bed) in an adjuvant setting might be optimal. However, it is avoided for post-wide local excision (oral tongue), as it can lead to toxicity. 


\section{Patients' selection, pre-treatment workup, and patients' care}

\section{Selection of patients for brachytherapy}

Selection of the patient for brachytherapy depends on following factors besides general status, type of cancer, and extent of disease:

- location;

- accessibility;

- $\operatorname{size}(<2 \mathrm{~cm}$ for radical curative BT);

- proximity to cartilage/bone (mandibular margin $>5 \mathrm{~mm}$ );

- presence or absence of trismus/submucous fibrosis (SMF);

- previous treatment;

- duration of previous treatment:

- gap between first treatment and relapse,

- dose details of the previous radiotherapy;

- suitability for general anesthesia for interstitial brachytherapy.

\section{Pre-treatment workup}

- Informed consent.

- Confirmation of diagnosis with histopathological details and stage the patient.

- Assessment of suitability of the patient for brachytherapy and selection of the intent.

- Illustration of the lesion with topography of disease based on clinical and imaging findings.

- Evaluation of the oral cavity for hygiene and dental carries, and its treatment in consultation with a dentist if necessary.

\section{Treatment strategy for head and neck brachytherapy}

The therapeutic aim/intent and indications for role of HDR brachytherapy in HNC depends on the stage of disease and treatment's classification as radical, adjuvant, boost, palliative, or salvage. HDR dose with respect to treatment intent, for a dose-rate of 4.5 Gy per fraction has been described. Brachytherapy clinician can choose different doses per fraction (3-5.5 Gy) or maintain the total dose or requisite biological equivalent dose (BED) with respect to therapeutic intent. The treatment intent and dose schedules have been illustrated in Figure 1.

Curative and radical: Early stage $\left(\mathrm{T}_{1-2} \mathrm{~N}_{0} \mathrm{M}_{0}\right)$ disease is considered for radical brachytherapy with curative intent, with HDR-BT of 45-54 Gy in 10-12 fractions, subject to tumor size. Tumors larger than $2 \mathrm{~cm}$, infiltrative with potential spread to lymph nodes, are deemed for a combination of EBRT radiation (including lymph nodes) with 40-46 Gy in 20-23 fractions. Brachytherapy boost of 16-26 Gy in 4-6 fractions is delivered in such cases with EBRT dose, with total BED of 85-90.

Adjuvant brachytherapy: Post-operative brachytherapy in the adjuvant setting may be used in case of close or positive margins, lymphatic invasion, or tumor infiltration $>5 \mathrm{~mm}$ in the absence of positive lymph nodes. The dose delivered is between 60-65 Gy in the adjuvant setting, following R0 resections, and between 10-25 Gy in the case of a local boost complementary to external beam therapy.

Potentially curative boost: Patients with primary (T1-2-3) disease can undergo the treatment, but the presence of lymph nodes $(\mathrm{N} 1-2 \mathrm{~b}-2 \mathrm{c}-0)$ or one $(<3 \mathrm{~cm})$ infiltration, requires taking care of involved node(s). In such cases, the primary lesion is treated with EBRT of 44-56 Gy (subject to tumor size) and neck nodes are treated with a radical dose of 66-70 Gy, depending on nodal size, with a simultaneous integrated boost (SIB) technique.

Salvage (recurrences including re-irradiation): Recurrence after previous radical treatment can be considered using BT alone or in combination with EBRT, subject to tumor size and interval from previous treatment and previous radiation doses, besides general and co-morbid medical status.

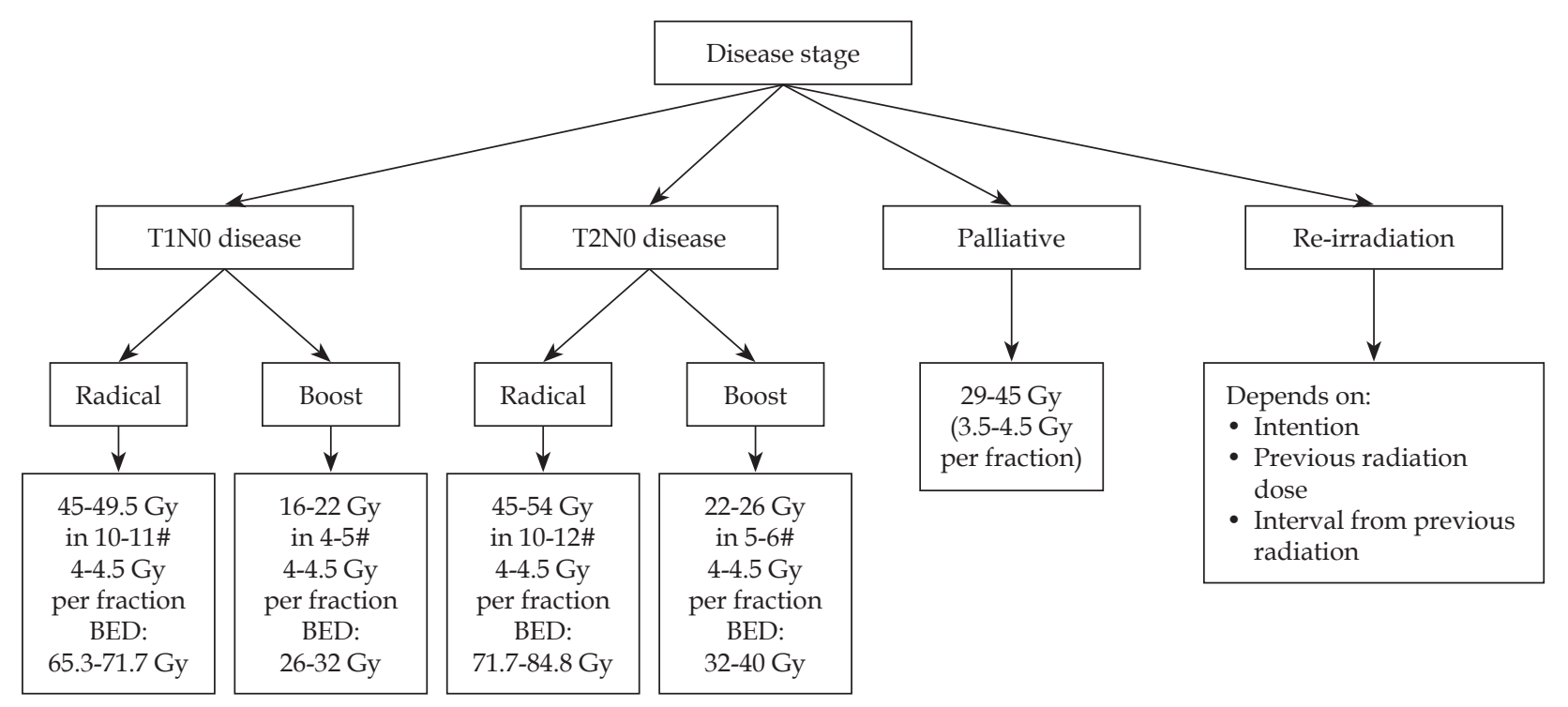

Fig. 1. Treatment intent and dose schedules in different stages of the disease 
Palliative: An advanced staged T4-2N2-3 cancer (with large primary and large node(s)) is generally treated with palliative intent. Small percentage of patients who present good response at the end or during the treatment both for primary and node(s), can be considered as good responders.

- These recurrences are not salvageable, but are treated with brachytherapy if there is no nodal or systemic relapse with a palliative dose of 29-45 Gy, with good growth constraint effect.

- Patients show better response (salvage) by delivering HDR-BT as a boost to the primary disease, after a gap of 6-8 weeks on an individual case-to-case basis, rather than awaiting a relapse. Here, the dose considered is 15-20 Gy, keeping BED up to about $115 \mathrm{~Gy}$.

\section{Anesthesia for head and neck brachytherapy}

- Anesthesia can be challenging in HNC brachytherapy patients. Preoperative assessment must be comprehensive, considering geriatric age group, difficult airway, and comorbid conditions must be highlighted.

- Standard pre-op guidelines: NPO 2 hours clear fluids and 6 hours light meal.

- A difficult airway cart must be kept ready at all times in the operating room. Awake intubation or intubation under general anesthesia can be decided individually as per anesthetist and institute protocol.

- Most of the patients will come under the category of anticipated difficult airway, therefore succinylcholine or non-depolarizing agent like rocuronium (if sugammadex is available) is preferred due to rapid onset and shorter duration of action.

- Post-intubation nasogastric tube insertion, before the execution of the procedure, for nutrition purpose (until removal of implant catheters) should be applied.

- Difficult extubation must be anticipated and difficult airway cart must maintain ready during extubation attempt. It is preferable to perform the extubation when the patient is fully awake and follow the instructions after adequate reversal. In some cases, the extubation may be postponed in view of airway edema, prolonged surgery, inadequate reversal, etc.

\section{Implant techniques}

- The procedure is carried out under general anesthesia.

- Mapping and proper documentation of local disease (topography) before treatment should be performed.

- Diagnostic images need to be maintained in the operating room, especially in case of a boost.

- All surgical instruments should be maintained as needed for head and neck (HN) surgery and specific for brachytherapy procedures, such as plastic/SS press buttons, silk threads, and electric cutter (for cutting the catheter keeping the dummy intact).

- Stocks of various catheters/tubes and needles should be kept, as per need of the implant site:

- single-lead catheter;

- single-lead with half/full moon button at other ends;

- double-lead catheter;

- single-lead with ' $\mathrm{T}$ ' ball catheter;
- 17 G SS needles having a bevel edge at one end (15 and $20 \mathrm{~cm}$ long).

- Prophylactic tracheostomy is not required for most HN sites, except for brachytherapy in post-pharyngeal wall and epiglottis lesions (SHSGL), where catheters are expected to interfere with smooth respiration because of special thyrohyoid approach by piercing through a thyrohyoid membrane, performed for optimum coverage of CTV [9].

- Inject initial dose of intravenous antibiotics and steroids (as per choice and hospital protocol).

- Insert feeding/Ryle's tube for nutritional support prior to starting the implant procedure.

- Place shoulder pad of requisite diameter under the shoulders and a rubber ring under the head (occiput) to allow easy maneuvering (ease of access for needle through submental skin) of the neck, as suitable to brachytherapist.

- Prepare the operative part and cover the body with sterile cloth, taking full aseptic precaution. Conduct a thorough clinical examination of the diseased organ and neck. Consider the submucosal extension of disease.

- Freehand insertion of 17 G SS needle(s) performed from submental area or as required by site, maintaining interline/ plane distance equal and parallel (1/1.4$1.6 \mathrm{~cm}$, average $1.5 \mathrm{~cm}$ ) as far as possible, keeping in mind surface/edge, where crossing the tissue for additional line is not possible.

- All subsequent needle(s) inserted as stated and as per the requirement of CTV/ITV in desired plane(s) and lines in each plane, as single, double plane, or volume implant (> 2 planes), and each time the needle is replaced by catheters.

- Check the patency of each catheter at the end of procedure to ease source movement. Secure the lower ends of catheters with half-moon button close to skin, but not too tight. Tally the lines in each plane(s).

- Isolate the thread (wound around the catheter underneath the bead/ball) of each line of the respective plane. Bind the threads of respective planes together. Now, club the bound threads of each plane and strap them together, and secure all of them on the side of the face without producing strain on catheters. Diagrammatic representation of the technique has been shown in Figure 2.

- Clean the oral cavity with saline using suction. Clean all the catheters protruding out from the submental surface and the skin puncture sites with spirit to remove blood/clot if any.

- The patient will remain in the recovery room till being weaned off from anesthesia for a couple of hours when he/she is ready for further steps of brachytherapy part of planning.

\section{Treatment planning and dosimetry}

\section{Treatment planning}

- Measurement of the catheters within and beyond the skin. 
- CT images taken with a slice thickness of 1-1.5 mm and transferred to the planning system for treatment planning (Figure 3).

- Dummy wires placed within the catheters helps to identify the individual catheter in the images, and the catheters can be individually tracked.

- Contouring of gross tumor volume (GTV)/clinical target volume (CTV) and critical organ at risk (OAR).

- Generation of virtual irradiation plan from loading of virtual source with respect to CTV.

- Evaluation of the isodose distribution from the generated plan and isodose line (dose-rate or prescription line) assessed, which encompasses desired CTV/internal target volume (ITV) without a cold or hot spot, providing dose inhomogeneity.

- In a case of oral tongue lesions, lateral edge and upper/superior surface are beneficial to under-dose. The upper or superior surface underdose can be resolved by using Bhalavat's T ball/bead technique. Lateral edge underdose, whenever required, can be settled by separate catheter or differential distance (between lines/planes nearer to shorter edge), compared to the one on opposite/away side or differential loading/ unloading of activity, or time which is expected to solve underdosage to some extent. Skin and subcutaneous dose is avoided or limited [20] (Figure 4).

\section{Target volume definition for brachytherapy}

- Gross tumor volume, CTV, and planning target volume (PTV) are three main volumes defined in radiotherapy planning for dose reporting. Additionally, ITV can be considered.

- Aim to treat adequately the CTV.
- For brachytherapy, CTV is considered the same as PTV.

- Gross tumor volume:

- definitive/radical treatment: clinical examination and imaging;

- boost/residual disease: clinical examination and post-radiation imaging;

- adjuvant: pre-surgery imaging and clinical imaging. Only CTV is taken into consideration.

- CTV: 0.5-1 cm (to account the tumor volume and anatomical barriers):

- lesion superficial vs. exophytic vs. infiltrative;

- residual disease and original GTV.
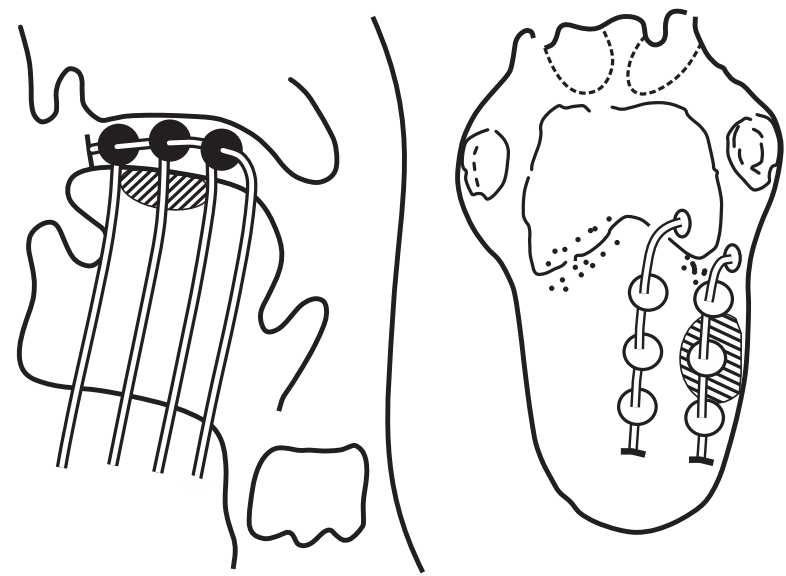

Fig. 2. Diagrammatic view of plastic bead/ball technique. The technique allows adequate coverage of the surface dose over the tongue
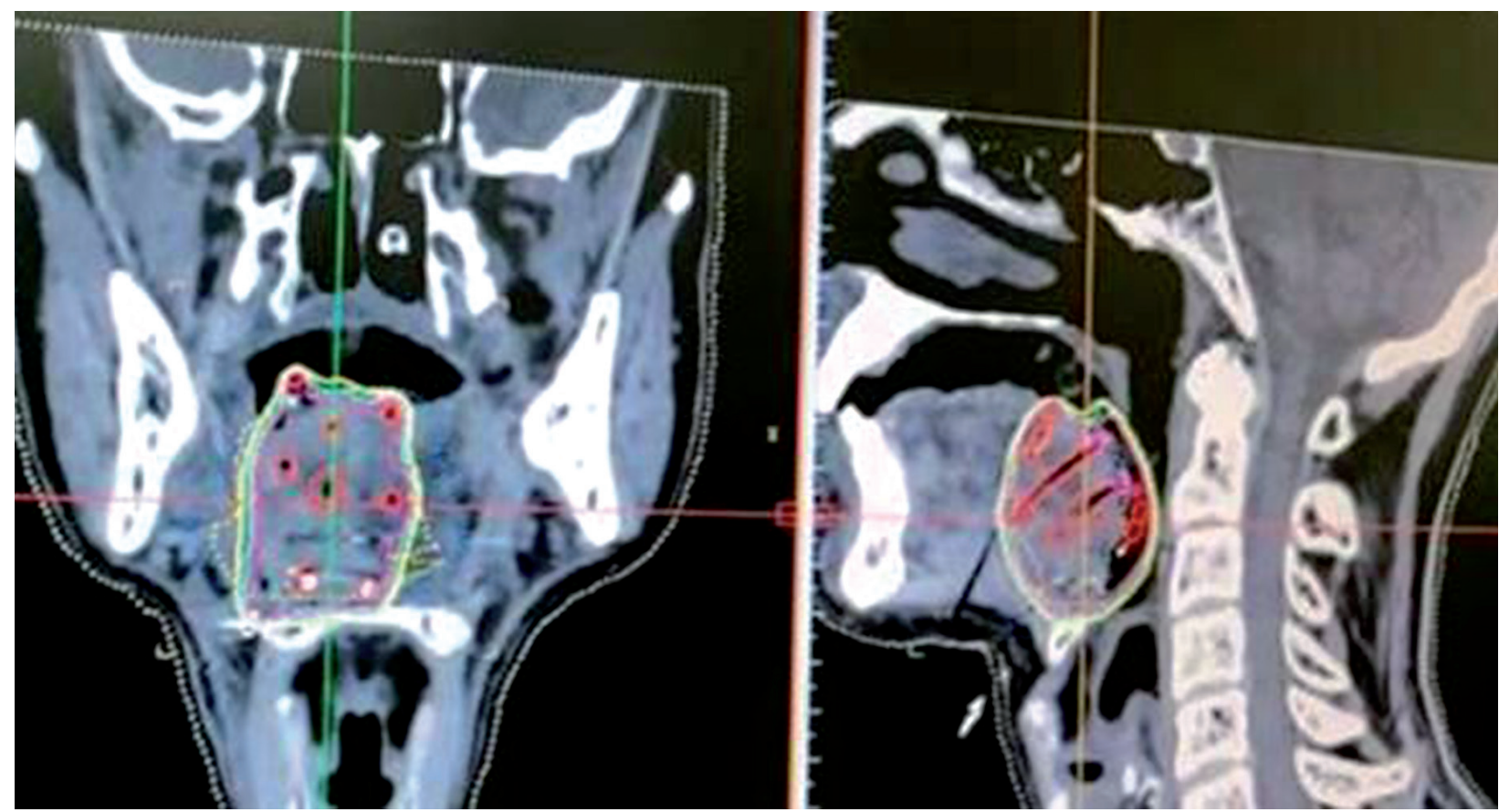

Fig. 3. Computerized tomography image of catheter implantation in base of tongue with overlay of dose distribution 


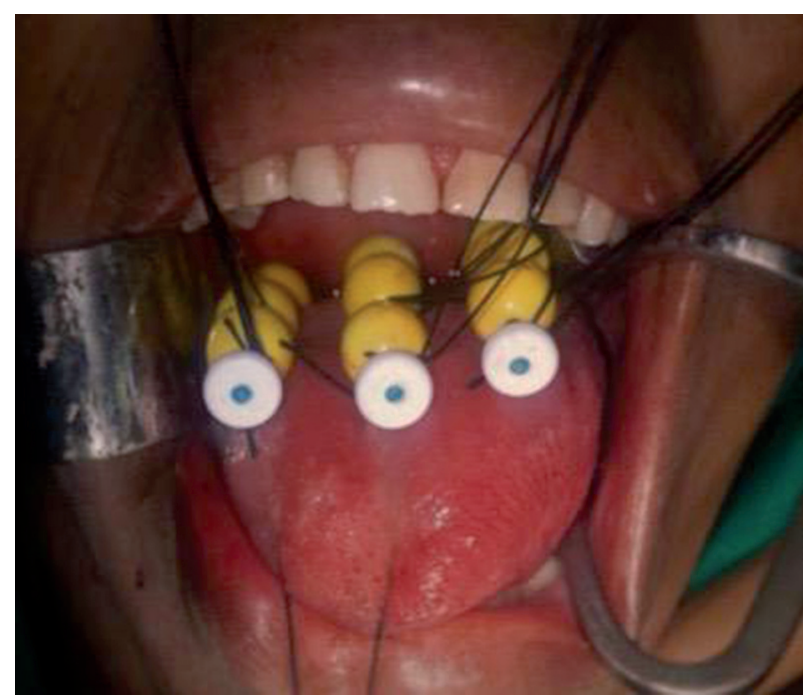

Fig. 4. Plastic bead placement with catheters in situ in multiplane implant using Bhalavat's technique

- ITV: Resolves peripheral fall off to deliver desired dose to measured CTV, and should be $0.5 \mathrm{~cm}$.

- Calculation of volume and decision of planes and lines for implant:

- create a virtual volume image of ITV, adding 3-5 mm margins to CTV;

- combine all lengths (length/breadth/thickness) of GTV as described above;

- plan the number of planes and lines in each plane needed to encompass CTV, keeping interline and plane distance of $1.4-1.6 \mathrm{~cm}$ and parallel to each other.

\section{HDR dose and dose-rate prescription}

The number of fractions for a planned total dose, calculated from a chosen dose per fraction (4-4.5 Gy), and delivered with two fractions per day not $<6$ hours apart. The details of doses are illustrated in Figure 1.

\section{Treatment planning and reporting}

- Description of clinical conditions, including GTV and CTV.

- Description of the technique (is the application performed following a system).

- Source specification, including reference air-kerma rate (RAKR) and total reference air-kerma (TRAK).

- A complete description of the time-dose pattern.

- Treatment description.

- Evaluation of mean central dose (MCD), minimum target dose, homogeneity index volumes, and their dimensions, including PTV/ITV, treated volume, high-dose regions, low-dose regions, reference volume, and irradiated volume (level 2).

- Coverage and conformity index.

- Dose non-uniformity ratio (DNR); ideally should be 0.35 .

- Dose for organs at risks (as followed for EBRT).

\section{Treatment monitoring and patient care during the implant}

This is as important as the execution of the procedure. Proper care of oral hygiene and tissue edema during treatment needs to be ensured. The use of mouth wash, analgesics, antibiotics, and steroids are necessary, and applied as needed pre-, post-, and during the procedure to keep oral cavity as clean as possible to prevent infection and tissue edema. Proper feeding is essential, which most cases is through a nasogastric tube and preferably a full diet. The use of shield for protection of OARs (mandible, palate) is required and the operator can take a call as per their experience. However, in HDR-BT, the use of cotton padding in proper location during delivery of radiation helps to reduce the OAR dose by way of distance and inverse-square law for the intensity of radiation to a certain extent.

\section{Removal of catheters}

Post-implant catheter removal is also as important as application, and is relatively a simple procedure if done meticulously. It is important to explain and counsel the patient about the removal procedure and how his co-operation will ease the removal of catheters. Implant catheters should be removed in well-lit operating room, under full aseptic conditions. Moreover, a monitor displaying the patient's vital statistics along with two attendants is required and one nurse to manage the emergency, and anesthetist, while dealing with oropharynx. Ideally, avoid catheter removal before 72 hours. Always keep saline and hydrogen peroxide bottle ready. Preferably, keep a few cups of a mixture of saline and hydrogen peroxide solution ready for use. Removal of catheters for the sites other than oral tongue and oropharynx is individual, from one end with a simple pull. For oral tongue and oropharynx, first pull each catheter about 5-10 mm and cut the catheters at inferior/submental end (at entry), so the length of each catheter within the tissue is smallest and allows easy removal. Removal can be done in two ways: one by one or by full assembly of catheters, together with one stroke of gentle pull. Steady removal to avoid brushing of the inflamed soft tissue should be performed, with respect to the implant site. The removed catheters are counted, and implant site inspected properly in order to make sure that all parts of caterers are removed. In case of bleeding during removal, maintain mouth opening with the use of retractor, and apply manual pressure (from inside and from the submental region); keep continuous pressure on bleeding side for about 5 minutes.

\section{Post-treatment patient care and follow-up}

After removal of all catheters, ask the patient to gargle with the mixture of saline and hydrogen-peroxide 3-4 times, and clean the oral cavity with the hand gently. The patient is normally discharged after the observation period of about 6-8 hours or the next day with details for precautions and follow-up explained. The nasal tube can be removed once the patient can easily swallow sips of water. The patient is requested to report in the case of 
bleeding from the implant site, excruciating pain, or any other significant complaint.

\section{Complications}

The rate of complications with brachytherapy has been reduced with the use of stepping source dosimetry system and the possibility of optimization. Most patients with brachytherapy may not experience radiation reaction during radiation period (3-6 days). They may have early signs of mucositis [21,22] by the time of catheters removal, but they might experience mucosal and/or skin reaction (grades II-III, rarely IV) depending on the location and volume irradiated by 3rd week, lasting for about 1-2 weeks and settling down to tolerable level, gradually resolving over the next 4-6 months. Occasionally, there can be a delay in healing and formation of ulcer, which may result with a fibrotic white scar. Radiation reaction healing is often delayed and requires attention and clinician care. The late effects, such as deep ulceration, necrosis, and occasionally, when close to cartilage or bone can cause cartilage necrosis and bone necrosis, respectively. However, the time or duration of the appearance, progress, and resolving of these conditions are not the same in all cases/ sites. It is not advised to take biopsies from such sites. Hypopigmentation, scarring, depigmentation, and cicatrization of the skin are the known late effects when the skin is treated $[23,24]$.

\section{Subsites for brachytherapy}

The various subsites in head and neck malignancies permit different modalities of brachytherapy, which include:

- interstitial brachytherapy (ISBT);

- endocavitary brachytherapy;

- surface mold.

\section{Interstitial brachytherapy}

Oral cavity, such as the lip, buccal tissues, floor of mouth, tongue, and hard palate, are the most accessible, and therefore the most suitable for brachytherapy. There is an extensive experience in LDR-BT treatment for almost all sites, with acceptable outcomes regarding various stages and complications [20,21,25,26,27], and can be extrapolated in terms of selection and indication for HDR-BT treatment, but it has not formed a part of care in its management. Applications and outcomes using HDRBT for selected cases, refused by patients, or not suitable for surgery, may show similar effectiveness. These tumors remain suitable even for surgery, particularly in their early stages. Small lesions of lip, FOM, and central and anterior (angle of mouth) of buccal mucosa (not involving alveolus/bone), oral tongue, and hard palate, are suitable for brachytherapy alone or as boost. It provides similar local control and survival rate, with the advantage of organ and function (speech and swallowing) preservation and better cosmesis [28].

Lip and buccal mucosa: Tumors more often present as early lesions, and those with a diameter $<5 \mathrm{~cm}$ can be exclusively treated with local BT + EBRT, with surgery in salvage setting. Nodal metastasis is low and can be considered for treatment when tumor size is more than $2 \mathrm{~cm}$ [7]. Radical brachytherapy is indicated for early lesions. Larger tumors $(>5 \mathrm{~cm})$ can be treated with EBRT, followed by brachytherapy. Early superficial lesions can also be treated with surface mold (single or sandwich) brachytherapy. In India, a large percentage of buccal mucosa cases present as gingiva-buccal complex and are thus treated with surgery. Patients with tumors that are anteriorly placed, involving the angle of the mouth and without involvement of superior and inferior gingivobuccal sulcus, are considered suitable for radical brachytherapy. Radical brachytherapy in these locations has the advantage of organ and function preservation, with excellent cosmetic outcomes in the majority of the patients $[29,30,31]$.

Oral tongue/floor of the mouth: Early (T1N0) lesions of both sites can be treated with curative intent with brachytherapy alone [32]. Addressing neck node is essential in patients with tongue cancer, as shown in a randomized trial [33], and can be approached by either EBRT or neck dissection at the time of brachytherapy. The sequence of EBRT and brachytherapy and its respective doses given may vary between different centers. However, we recommend performing brachytherapy prior to EBRT and doses as mentioned above.

Oropharynx: A combined EBRT + BT approach is recommended for oropharyngeal cancers in view of a high incidence of ipsilateral and bilateral nodal metastases even in early lesions [34]. Lateralized T1 tumors of the tonsil are considered for brachytherapy alone. Substantial morbidity by surgery, carried out as an early primary management for oropharynx, is now been replaced by EBRT with or without concurrent chemotherapy in the last 4-5 decades for most stages, with brachytherapy as a boost for suitable stage $[35,36,37]$.

Epiglottis (SHSGL) involvement from vallecula/BOT is frequent, and if the free edge of epiglottis laryngeal and/ or lingual surface is involved, infra-hyoid approach is indicated for brachytherapy for the posterior plane, which is passed through thyro-hyoidal membrane to resolve the laryngeal surface of epiglottis. Epiglottis lesion, as alone, is infrequently seen and can be managed in similar lines [38].

\section{Endocavitary brachytherapy}

Nasopharynx (NPC) and external auditory canal (EAC) are the sites having natural tubular space, in which radioactive source can be placed to deliver radiation around the placed source. The addition of intraluminal/cavitary BT (ICBT/ILRT/INRT) boost to the total dose around the nasopharynx to improve local control have been well described [39]. Most major centers around the world, experienced in treating large numbers of patients with NPC, have advocated the use of involved-node radiotherapy (INRT). With increasing experience of INRT, this technique has been added as a boost for early $\mathrm{T} 1 / 2$ tumors treated with radical intent and as salvage therapy for recurrences. Patients with radical intent receive 70-76 Gy of prescribed dose delivered with conventional methods, and EBRT is delivered 
with 46-56 Gy in 23 to 28 fractions over 4-6 weeks, followed by INRT with $10-15$ Gy in 2/3 fractions. Palliative treatment for recurrence can be given in 3-4 fractions of 5 Gy each, depending on the size and type of lesion [40]. Lesions, which are superficial and $<4 \mathrm{~mm}$ in depth are ideal for such a treatment.

Nasopharynx: INRT requires special applicator and steps for application, which are described in detail in Table 2. It is found to be safe and well-tolerated, with

Table 2. Steps of nasopharyngeal brachytherapy and dosimetry (INRT)

- Material required: TWO (2) infant feeding tube (IFT) (7 fr), Rotterdam's nasopharyngeal brachytherapy applicator (RNPBA), silk thread roll (2-0), lignocaine jelly/spray for local anesthesia

- Preparation of IFT:

- Prepare two IFT with silk thread within and protruding out enough at both the ends. Pass long silk threads through both infant feeding tubes

- Spray lignocaine in patient's mouth, nose, and pharyngeal region

- Patient in supine position. Spread sterile towel over patient's neck and chest

- First step: Inserting IFT in each nasal cavity:

- Pass the closed end of threaded IFT into the nasal cavity (controlling the thread at another end) and take it out through the oral cavity, one by one (make sure to keep the tubes with threads separate on each side)

- Second step: Preparation of RNPBA:

- Concave side of applicator should face to air/above

- Allow the closed end with thread of the IFT (oral end) to pass through relevant long limb of RNPBA (right for right and left for left) and bring it out from the other end, such that the closed end (oral end) of IFT with thread is seen protruded out from smaller limb of RNPBA

- Procedure repeated for another limb of RNPBA

- Tie the loose end of both threads together tightly, close to the cut surface of IFT

- Third step: Placing RNHBA, such that it lies apposite to nasopharynx:

- Pull the nasal ends of IFT simultaneously and allow the RNHBA to pass through oral cavity (gentle push of applicator from below if required) and behind the soft palate in such a way that body of the RNHBA settles and closely affixed to the nasopharynx roof

- See and ask if the patient is comfortable with the application

- Once it is all set and the patient is comfortable, remove the IFT through the nasal cavity (knot at the end of threads helps the thread/ applicator to stay in place as intended)

- Insert the stopper at the free nasal end of applicator close to the nasal opening

- Take the planning CT scan with applicator and a dummy

- Track the dummy and create virtual plan, in which planned surface/ area of nasopharynx receives desired dose. Prescription at 2-3 $\mathrm{mm}$ from the surface, where $D_{200}(2 R D R)$ is within tube and $D_{150}$ is close to it few side effects. Xerostomia is noted universally in all patients and is attributed to EBRT. Chronic serous otitis media is observed in about $20 \%$ of patients, whereas selflimiting epistaxis is noted in a few patients. Complications of treatment more likely due to INRT, such as nasal mucosal synechiae and ulceration, are observed occasionally, although these are largely asymptomatic.

External auditory canal: The external ear consists of the pinna (auricle) and EAC, and both are treated with surface mold and intracavitary brachytherapy, respectively, depending on type and stage of the lesion. Early stage EAC can be treated with radical treatment, with the combination of external beam radiation therapy and BT (ICRT) as modality of choice for its efficacy, resulting in less severe side effects and better cosmetic outcomes [41].

\section{Surface mold (SM) in HNC}

Small, non-infiltrative mostly superficial (> $5 \mathrm{~mm}$, thickness) lesions of the skin of face, including the nose and pinna of the ear as well as mucosal surface of accessible sites, such as oral cavity (lip, buccal mucosa, and hard palate), can be considered for SM brachytherapy. A mold, using flexible source of ${ }^{192} \mathrm{Ir}$ (previously LDRBT, now HDR-BT), which has overcome the accessibility problem of cone applicator, needs to deliver radiation with an advantage of controlling dose to bone/cartilage close to lesion by way of its property of peripheral fall-off. It is useful as primary or even as salvage modality, with excellent patients' compliance and disease control [10]. Surface carrier prosthesis, which fits the target region, needs to be prepared. The process is explained in Table 3 .

Table 3. Steps of surface mold brachytherapy

Preparation of surface carrier prosthesis (SCP)

- Material required: Dental wax bolus, strips of plaster of Paris bandage/stone plaster/acrylic powder

- Take an impression of the lesion and its surrounding surface or area with the help of strips of plaster of Paris or dental compound. Prepare the model of that part by pouring/layering fluid plaster of Paris or stone plaster onto or into the impression

- SCP with respect to location/site is constructed on this model using acrylic powder or Perspex and the SCP is fitted with lesion

- Lesion's impression is copied onto the side of SCP, close to lesion, or else, one should draw the lesion using patent blue ink to visualize a virtual image of lesion on the SCP

- Computerized tomography or orthogonal X-ray film of the area under treatment is taken with SCP in-situ

- HDR planning:

- GTV should be covered by $90-95 \%$ of isodose and PTV by $85-90 \%$ of isodose

- Dose is prescribed to the highest isodose covering almost the entire desired GTV

- The higher dose should remain within the mould - Delivery of number of fraction, for total dose, decided from chosen dose per fraction and delivered twice daily at 6 hours interval 


\section{Role of brachytherapy in re-irradiation}

\section{Re-irradiation background}

In spite of many advances in the management of head and neck malignancies, approximately $20-50 \%$ of patients are diagnosed with a loco-regional recurrence within the first two years. Surgical salvage is the major treatment modality, but is possible in only $20 \%$ of patients, leading to an overall 5-year survival of $20-30 \%$ [42]. Re-irradiation using EBRT can lead to severe local toxicities. HDR-BT can achieve a high-dose directly to the target volume and provides the advantage of a rapid dose fall-off, thereby, allowing for sparing of normal tissues [43]. HDR-BT offers significant advantages over conventional LDR brachytherapy by reducing the concerns of radiation safety for the staff during hospitalization and provides the clinician with greater control over the dose distribution by using computer-optimized dwell times within the individual catheters. With HDR-BT, dosimetrically equivalent or superior outcomes compared to LDR brachytherapy for gynecological tumors and other locations have been previously achieved.

\section{HDR brachytherapy in re-irradiation}

The prescribed dose is in the range of 3.5-4.5 Gy per fraction, depending on the site and status of the disease. The implant tubes are removed after the planned BT doses are delivered. Total dose (EBRT-BT) is kept within tolerance level and is assessed by estimating biologically equivalent doses (BED). The dose with radical BT is 40.5 Gy and the dose with BT boost is $27 \mathrm{~Gy}$. In a series of twenty-five patients [44] treated with re-irradiation in recurrent head and neck malignancies, the median survival calculated for patients who underwent re-irradiation with HDR-BT at 1- and 2-year overall survival was $77 \%$ and $68 \%$, respectively, with a median follow-up of 25 months.

\section{Recent guidelines}

The latest report by GEC-ESTRO has reported on the role of HDR-BT as a salvage option in re-irradiation in the head and neck malignancies [7]. For a patient, who is ineligible for surgical salvage, brachytherapy is an acceptable option, provided adequate CTV coverage with no significant bone invasion, fistula, or limited life expectancy. Brachytherapy in previously full course irradiated regions, needs to follow the same principles as primary brachytherapy with strict dose and volume constraints. Additionally, interstitial brachytherapy can play an important role in the treatment of lymph node recurrences of head and neck cancer. Using image-guided interstitial HDR-BT or LDR-BT for re-irradiation of recurrent lymph node metastases of head and neck cancer, local control probabilities in the range of approximately $60-70 \%$ have been published $[45,46,47,48]$.

\section{Discussion}

Head and neck site is the most complex and challenging anatomic site, especially where basic physiological function of deglutition, phonation, hearing, vision, and breathing are associated with the subsites. Moreover, there is a need for protection and preservation of the site, while considering its treatment by any modality. Therefore, a treatment modality, which reduces treatment-related toxicity and offers better post-treatment QoL is required to provide a balanced therapeutic ratio [49]. Surgery, external radiation, brachytherapy, or combination with or without chemotherapy, remains the treatment modality of various sites in head and neck malignancies. Despite advancement in its management by different modalities, loco-regional failure is observed in approximately $20-50 \%$ and remains the major cause of poor survival, rather than a systemic failure. There is a need for intensification of dose for higher local control and survival. It is possible to deliver a higher dose by brachytherapy and sparing surrounding organs at risk with limited morbidity. Classical indications for brachytherapy in HNC patients are the oral cavity, oropharynx, and nasopharynx, epiglottis with an interstitial method of implant and skin, nasal cavity, and external auditory canal with surface mold or intraluminal method of application. Oral cavity and oro-pharynx produce almost $60 \%$ of HNC [1] and are the sites, which are the most suitable for brachytherapy application. Brachytherapy requires an initial learning curve, such as surgery. The procedure requires special skills and continuous training, including the ability to organize, and some of the procedures need multidisciplinary applications with a high level of expertise. Head and neck brachytherapy is not being practiced due to complex anatomical shapes, the curvature of head and neck site, and complications associated with brachytherapy application and its dosimetry.

Overall, brachytherapy is an important part of radiation therapeutic armamentarium and classic indication for both primary and recurrent squamous cell carcinoma of the HNC. The majority of available data on brachytherapy is on LDR-BT techniques. In terms of indication, the application of HDR-BT is no different from LDR-BT. Total dose for HDR-BT has been derived from LQ model, using a mathematical model, which is comparable to LDR-BT and conventional EBRT, and is delivered in a number of fractions with respect to selected dose per fraction twice daily, with an interval of at least 6 hours. Computer-based stepping source technology allows optimization of dose distribution based on 3-D imaging, which facilitates the calculation of dose from the dose-volume histogram (DVH) for CTV and OAR, thereby allowing for a comparison with other centers. It needs to be remembered that there is limited number of experiences with HDR brachytherapy in patients with head-and-neck cancers in India and even abroad. Therefore, some of these suggested doses have not been extensively tested in clinical practice. Any clinician following these guidelines is expected to use clinical judgment to determine an individual patient's treatment.

\section{Conclusions}

Brachytherapy in HNC is an excellent treatment modality providing the best outcomes regarding local control and reduced toxicities. The IBS hopes to encourage and enhance the interest of students of radiation 
oncology, and as for young beginners, to adopt the role of brachytherapy in their clinical practice as well as advanced EBRT technology and its appropriate combinations. Randomized trials need to be planned in this regard, considering the need for uniformity in treatment planning and dose delivery, as these guidelines can help to design the future trials in head and neck malignancies. The IBS and authors of the article hope that these guidelines and recommendations will rejuvenate the interest in brachytherapy for the HNC lesions among all the members of the radiation oncology society.

\section{Disclosure}

The authors report no conflict of interest.

\section{References}

1. Bray F, Ferlay J, Soerjomataram I et al. Global cancer statistics 2018: GLOBOCAN estimates of incidence and mortality worldwide for 36 cancers in 185 countries. CA Cancer J Clin 2018; 68: 394-424.

2. Mishra A, Meherota R. Head and neck cancer: global burden and regional trends in India. Asian Pac J Cancer Prev 2014; 15: 537-550.

3. Ash D, Gerbaulet A. Oral tongue cancer. In: GEC ESTRO Handbook of Brachytherapy, 2002; pp. 237-251.

4. Mazeron JJ, Ardiet JM, Haie-Meder C et al. GEC-ESTRO recommendations for brachytherapy for head and neck squamous cell carcinomas. Radiother Oncol 2009; 91: 150-156.

5. Harréus U. Surgical errors and risks - the head and neck cancer patient. GMS Curr Top Otorhinolaryngol Head Neck Surg 2013; 12.

6. Kouloulias V, Thalassinou S, Platoni K et al. The treatment outcome and radiation-induced toxicity for patients with head and neck carcinoma in the IMRT era: a systematic review with dosimetric and clinical parameters. Biomed Res Int 2013; 2013: 401261.

7. Kovács G, Martinez-Monge R, Budrukkar A et al. GEC-ESTRO ACROP recommendations for head \& neck brachytherapy in squamous cell carcinomas: 1st update - Improvement by cross-sectional imaging-based treatment planning and stepping source technology. Radiother Oncol 2017; 122: 248-254.

8. Erickson BA, Demanes DJ, Ibbott GS et al. American Society for Radiation Oncology (ASTRO) and American College of Radiology (ACR) practice guideline for the performance of high-dose-rate brachytherapy. Int J Radiat Oncol Biol Phys 2011; 79: 641-649.

9. Peiffert D, Castelain B, Thomas L et al. Pulsed dose rate brachytherapy in head and neck cancers. Feasibility study of a French cooperative group. Radiother Oncol 2001; 58: 71-75.

10. Strnad V, Lotter M, Grabenbauer G et al. Early results of pulsed-dose-rate interstitial brachytherapy for head and neck malignancies after limited surgery. Int J Radiat Oncol Biol Phys 2000; 46: 27-30.

11. Koedooder K, Van Herten Y, Van der Grient HN et al. Safety aspects of pulsed dose rate brachytherapy: analysis of 1225 patients. Nowotwory. I Oncol 2007; 57: e230-232.

12. Melzner WJ, Lotter M, Sauer R et al. Quality of interstitial PDR-brachytherapy-implants of head-an-neck cancers: Predictive factors for local control and late toxicity? Radiother Oncol 2007; 82: 167-173.

13. Harms W, Peschke P, Weber KJ et al. Dose-dependent differential effects of low and pulsed dose rate brachytherapy in a radioresistant syngeneic rat prostate tumor model. Int J Radiat Biol 2002; 78: 617-623.
14. Pernot M, Malissard L, Aletti P et al. Iridium-192 brachytherapy in the management of $147 \mathrm{~T} 2 \mathrm{~N} 0$ oral tongue carcinomas treated with irradiation alone: comparison of two treatment techniques. Radiother Oncol 1992; 23: 223-228.

15. Wendt CD, Peters LJ, Delclos L et al. Primary radiotherapy in the treatment of stage I and II oral tongue cancers: importance of the proportion of therapy delivered with interstitial therapy. Int J Radiat Oncol Biol Phys 1990; 18: 1287-1292.

16. Bourgier $C$, Coche-Dequéant $B$, Fournier $C$ et al. Exclusive low-dose-rate brachytherapy in 279 patients with T2N0 mobile tongue carcinoma. Int J Radiat Oncol Biol Phys 2005; 63: 434-440.

17. Mazeron JJ, Grimard L, Raynal M et al. Iridium192 curietherapy for T1 and T2 epidermoid carcinomas of the floor of mouth. Int J Radiat Oncol Biol Phys 1990; 18: 1299-1306.

18. Mazeron JJ, Simon JM, Le Péchoux C et al. Effect of dose rate on local control and complications in definitive irradiation of T1-2 squamous cell carcinomas of mobile tongue and floor of mouth with interstitial iridium-192. Radiother Oncol 1991; 21: 39-47.

19. Mendenhall WM, Parsons JT, Stringer SP et al. T2 oral tongue carcinoma treated with radiotherapy: analysis of local control and complications. Radiother Oncol 1989; 16: 275-281.

20. Marinello G, Pierquin B. The Paris system, optimization of dose, and calculation of treatment time. In: Pierquin B, Marinello G (eds.). A practical manual of brachytherapy. Medical Physics Publishing, Madison 1997; pp. 53-61.

21. Sasaki S, Teshima T, Murayama S et al. Comparison of radiation mucositis after interstitial brachytherapy between LDR and HDR. Head Neck Cancer 1993; 19: 197-200.

22. Urashima Y, Nakamura K, Shioyama Y et al. Long term functional outcome of brachytherapy for carcinoma oft he mobile tongue: focus on atrophic chance of irradiated tongue. Jpn J Clin Oncol 2006; 36: 681-687.

23. Pernot M, Luporski E, Hoffstetter $\mathrm{S}$ et al. Complications following definitive irradiation for cancers oft he oral cavity and the oropharynx (in a series of 1134 patients). Int J Radiat Oncology Biol Phys 1997; 37: 577-585.

24. Mazeron JJ, Simon JM, Le Pechoux C et al. Effect of dose rate on local control and complications in definitive irradiation of T1-2 squamous cell carcinomas of mobile tongue and floor of mouth with interstitial iridium-192. Radiother Oncol 1991; 21: 39-47.

25. Fijuth J. Is there any place for LDR brachytherapy for head and neck carcinomas in HDR era? J Contemp Brachytherapy 2009; 1: 62-66.

26. Inoue Ta, Inoue To, Yoshida K et al. Phase III trial of high vs. LDR interstitial radiotherapy for mobile tongue cancer. Int J Radiat Oncol Biol Phys 2001; 51: 171-175.

27. Ayerra AQ, Mena EP, Fabregas JP et al. HDR and LDR Brachytherapy in the Treatment of Lip Cancer: the Experience of the Catalan Institute of Oncology. J Contemp Brachytherapy 2010; 2: 9-13.

28. Yamazaki H, Yoshida K, Yoshioka Y et al. High dose rate brachytherapy for oral cancer. J Radiat Res 2013; 54: 1-17.

29. Pathak KA, Nason R, Talole $S$ et al. Cancer of the buccal mucosa: a tale of two continents. Int J Oral Maxillofac Surg 2009; 38: $146-150$

30. Orecchia R, Rampino M, Gribaudo S et al. Interstitial brachytherapy for carcinomas of the lower lip. Results of treatment. Tumori 1991; 77: 336-338.

31. Tombolini V, Bonanni A, Valeriani M et al. Brachytherapy for squamous cell carcinoma of the lip. The experience of the Institute of Radiology of the University of Rome 'La Sapienza'. Tumori 1998; 84: 478-482.

32. Marsiglia H, Haie-Meder C, Sasso G et al. Brachytherapy for T1-T2 floor of mouth cancers. The Gustave-Roussy Institute experience. Int J Radiat Oncol Biol Phys 2002; 52: 1257-1263. 
33. D'Cruz AK, Vaish R, Kapre $\mathrm{N}$ et al. Elective versus therapeutic neck dissection in node negative oral cancer. N Engl J Med 2015; 373: 521-529.

34. Inoue $T$, Inoue $T$, Teshima $T$. High dose rate interstitial brachytherapy for mobile tongue cancer: part 1. Phase I/II study of HDR hyperfractionated interstitial brachytherapy for oral cancer. Jpn J Cancer Chemother 2000; 27: 287-290.

35. Hofstetter S, Marchal C, Peiffert D et al. Treatment duration as a prognostic factor for local control and survival in epidermoid carcinoma of the tonsillar region treated by combined external beam irradiation and brachytherapy. Radiother Oncol 1997; 45: 141-148.

36. Levendag P, Nijdam W, Noever I et al. Brachytherapy versus surgery in carcinoma of tonsillar fossa and/or soft palate: late adverse sequelae and performance status: can we be more selective and obtain better tissue sparing? Int J Radiat Oncol Biol Phys 2004; 59: 713-724.

37. Meyer JE, Brocks C, Gehrking E et al. Brachytherapy in combination with function-preserving surgery. An interdisciplinary challenge. HNO 2008; 4: 471-478.

38. Bhalavat RL, Pathak KA, Mahantshetty U et al. Brachytherapy boost: A novel approach for epiglottic carcinoma. Brachytherapy 2007; 6: 212-217.

39. Levendag PC, Keskin-Cambay F, de Pan C et al. Local control in advanced cancer of the nasopharynx: Is a boost dose by endocavitary brachytherapy of prognostic significance? Brachytherapy 2013; 12: 84-89.

40. Fischer M, Stüben G, Stuschke M et al. Brachytherapy with 192 Iridium in the treatment of recurrent nasopharyngeal carcinoma. Laryngorhinootologie 2002; 81: 106-110.

41. Budrukkar A, Dasgupta A, Pandit P et al. Clinical outcomes with high-dose-rate surface mould brachytherapy for intraoral and skin malignancies involving head and neck region. I Contemp Brachytherapy 2017; 9: 242-250.

42. Hamoir M, Schmitz S, Suarez C et al. The current role of salvage surgery in recurrent head and neck squamous cell carcinoma. Cancers (Basel) 2018; 10: 267.

43. Krempien RC, Grehn C, Haag C et al. Feasibility report for treatment of locally recurrent head-and-neck cancers by combined brachy-chemotherapy using frameless image-guided 3D interstitial brachytherapy. Brachytherapy 2005; 4: 154-162.

44. Bhalavat R, Pareek V, Chandra M et al. High-dose-rate interstitial brachytherapy in recurrent head and neck cancer: an effective salvage option. J Contemp Brachytherapy 2018; 10: 425-430.

45. Narayana A, Cohen G, Zaider M et al. High-dose-rate interstitial brachytherapy in recurrent and previously irradiated head and neck cancers - preliminary results. Brachytherapy 2007; 6: 157-163.

46. Puthawala A, Syed NAM, Gamie S et al. Interstitial lowdoserate brachytherapy as a salvage treatment for recurrent headand-neck cancers: Long-term results. Int J Radiat Oncol Biol Phys 2001; 51: 354-362.

47. Tselis N, Ratka M, Vogt HG et al. Hypofractionated accelerated CT-guided interstitial 192Ir-HDR brachytherapy as re-irradiation in inoperable recurrent cervical lymphadenopathy from head and neck cancer. Radiother Oncol 2010; 98: 57-62.

48. Rudzianskas V, Inciura A, Juozaityte E et al. Reirradiation of recurrent head and neck cancer using high-dose-rate brachytherapy. Acta Othothinolaryngol Ital 2012; 32: 297-303.

49. Bajwa HK, Singareddy R, Alluri KR et al. High-dose-rate interstitial brachytherapy in oral cancer - its impact on quality of life. Brachytherapy 2016; 15: 381-386. 